discussion, which dealt, among other things, with the guilt feelings of Britons and Americans at not being. adequately prepared for Nazi or Japanese aggression, with the fact that the chosen seapegoat need not necessarily be innocent as judged by rational standards, and with the possible existence of rudimentary guilt and scapegoatism in animals.

\section{THE ATOMIC BOMB}

A PAMPHLET, "Statements relating to the Atomic Bomb", published by H.M. Stationery Office (price 4d. net), gives some account of the activity in Great Britain and the United States leading to the use of this weapon in Japan by the United States Army Air Force. Reference is made to statements issued by the United States and Canadian Governments giving an account of the work carried out in these countries which led up to or was associated with the achievement, and an outline of the scientific background. The statement includes a brief summary of earlier work on nuclear disintegration which bears only indirectly on the phenomenon of nuclear fission, on which the activity of the atomic bomb depends, and then gives some account of the work on the fission of uranium nuclei, published prior to the beginning of the War, and the development of which on a large scale led to the atomic bomb.

In 1934 Fermi and his colleagues in Rome subjected uranium to neutron bombardment and showed that new isotopes were formed which were radioactive, and it was thought that atoms of atomic number higher than 92, that of uranium, had been produced. These elements were not known in Nature. In 193839, however, Hahn and Strassmann, in Berlin, made experiments which showed that one, at least, of the new isotopes believed to be of higher atomic number and mass than those of uranium was really an isotope of barium, which had an atomic number and mass about half that of uranium. Frisch and Meitner thereupon pointed out that a phenomenon which they called 'nuclear fission' had occurred, the uranium nucleus being split into two parts of roughly equal mass, and that the two parts of the divided uranium nucleus should fly apart with great energy, a prediction verified experimentally by Frisch in Copenhagen. Confirmation of this fission process, and of the great liberation of energy, was independently obtained by Joliot in Paris, and by many other physicists.

Early in 1939, Joliot, Halban and Kowarski proved experimentally what had been predicted theoretically, that in the fission of uranium, a number of free neutrons were also produced, and independent confirmation was obtained by Anderson, Fermi, Hanstein, Szilard and Zinn in the United States. It was clear that the fission not only provided a large amount of energy by reason of the loss of total mass which had occurred, according to Einstein's theory of the correlation of mass and energy, but also that the liberation of neutrons in the process, which was produced by neutron bombardment, suggested that each time a uranium nucleus underwent fission, the reaction might be accelerated by the neutrons which it itself produced. It would, in fact, be analogous in the field of nuclear chemistry to the well-known chain reactions in the chemistry of stable atoms and mole. cules. The process could be started by the application of only a minute fraction of the energy which would ultimately be liberated.
Bohr and Wheeler had published a theory of the fission process in 1939 in which it appeared that the isotope of mass 235, present in only small amount in natural uranium, which consists mostly of the isotope of mass 238, should undergo fission particularly easily when the bombarding neutrons had a very low energy, whereas the isotope of mass 238 requires high-energy neutrons. This prediction was verified in 1940 by Nier, and by Booth, Dunning and Grosse, in the United States. Neutrons of an intermediate energy-range are strongly absorbed by the uranium 238 nucleus; but instead of producing fission, a new nucleus of mass 239 is formed. This has the property of emitting two electrons in succession, thus forming nuclei of atomic numbers 93 and 94, which do not occur in Nature. The nucleus of atomic number 94 should be capable of undergoing fission with the greatest ease when bombarded with neutrons of very low energy. The elements of mass 235,238 and 239 are not the only ones which should undergo fission; thorium should undergo fission with bombarding neutrons of very high energy, and protactinium (mass 231, atomic number 91) should behave in a way intermediate between uranium 235 and 238.

The energy liberated in uranium fission is stated to be millions of times greater than that set free in the combustion of an equal weight of oil or coal; but the process must obviously be controlled, and a suitable 'slowing-down' medium is necessary, so that fast neutrons produced by fission would lose their energy by elastic collisions before producing further fission. Such a medium is provided by heavy water, or deuterium oxide. In an atomic bomb a quantity of uranium 235 greater than a certain critical amount is necessary. This is because the reaction depends on the conservation of the neutrons produced by the fissions. In a block of uranium, the proportion of neutrons which escape into the outer air, and thus become ineffective, is reduced by increasing the size of the block, and explosion is possible only with a certain minimum amount of material. Quantities less than this are perfectly safe. Thus, it is only necessary to bring together two pieces each less than the critical size, but exceeding it when in contact, to detonate the bomb. It is stated that the tempera. ture of the mass then reaches many million degrees and the $p$ ressure many millions of atmospheres. The reaction must develop so rapidly that a substantial part of the material can react before the system has time to fly apart, and the neutrons produced in the fission process are fast enough to fulfil this condition, unless they are slowed down by artificial means.

Details are given of work at Liverpool and Cambridge on the dimensions of the bomb and related problems, and on the method of separation of the uranium 235 isotope by a gaseous diffusion method at Oxford and Birmingham. During this period, similar problems were occupying American scientific men. In 1942, it is stated, it became clear that the scale upon which research and development could be underteken in the United Kingdom must be far smaller than in America. In 1943 a large research estrablishment was set up in Montreal, Canada, and in 1944 a British-Canadian-American project was in operation near Petawawa, Ontario.

The report gives the constitution of many com. mittees set up, and of Government actions at various stages, and is a record of well-co-ordinated and actively prosecuted effort in the many directions necessary before the atomic bomb became a reality. 